\title{
Graphene encapsulation enables vitreous ice sample for APT and near-atomic reconstruction of nanoparticle-liquid interface
}

Shi Qiu ${ }^{1}$, Gediminas Gervinskas ${ }^{2}$, Hariprasad Venugopal ${ }^{2}$, Ross Marceau ${ }^{3}$ and Jing Fu ${ }^{1}$

${ }^{1}$ Monash University, Clayton, Victoria, Australia, ${ }^{2}$ Monash University, Victoria, Australia, ${ }^{3}$ Deakin University, Geelong, Victoria, Australia

We report an approach based on graphene encapsulation to investigate the structure and chemical composition of liquid samples with APT. The specimens for probing are prepared by encapsulating the solution with a single graphene membrane on top of a W or Si specimen needle tips, with the final diameter maintained at less than $100 \mathrm{~nm}$. The liquid specimens are then transferred to a laser-pulsed atom probe instrument, and frozen by direct loading and contact onto the cryogenically cooled stage. Field evaporation has been proven to be feasible, and the mass-to-charge-state ratio spectrum acquired from APT determines the ion species. The reconstructed 3D chemical maps further reveal the original position of ions within the frozen hydrated sample [1]. This approach allows exploring domains previously unreachable, such as a single protein in solution [2].

To confirm the sample preparation process, additional graphene-encapsulated liquid specimens have been directly loaded to the cryogenic stage in cryo-TEM without any pre-freezing technique, which is similar to the cooling method performed in the APT experiment. The TEM image acquired, as well as the diffraction pattern (Figure 1), reveal that no significant crystalline ice has been formed in the sub-100 nm frozen hydrated domain. This suggests contact freezing of graphene-encapsulated liquid specimens is an effective approach to ensure the near-native state of frozen samples. Using gold nanoparticles (AuNPs) in suspension as an example, analysis of the mass spectrum and three-dimensional (3D) chemical maps (Figure 2) from APT provides a detailed image of the water-gold interface at near-atomic resolution [3]. A locally dense region of $\mathrm{Au}^{+}$ions has been reconstructed, representing a portion of an individual AuNP. A large number of water-related ions have also been identified, confirming the AuNP in the hydrated state. At the water-gold interface, the trisodium-citrate layer has been observed based on $\mathrm{Na}^{+}$and C-containing ions.

Acknowledgement.

The authors acknowledge funding from the Australian Research Council (DP180103955) and also the contributions from coauthors in [1-3]. This work was performed in part at the Melbourne Centre for Nanofabrication (MCN), Victorian Node of the Australian National Fabrication Facility (ANFF). Also, the authors acknowledge the use of facilities within the Monash Centre for Electron Microscopy (MCEM), Monash Ramaciotti Cryo-EM platform, Monash Campus Cluster (MCC), and Deakin University's Advanced Characterisation Facility. 



Figure 1. (a) Cryo-TEM image of graphene-encapsulated solution for APT on the pre-sharpened TEM half grid. (scale bar: $100 \mathrm{~nm}$ ) (b) Diffraction pattern acquired from the region of frozen solution (scale bar: $5 \mathrm{~nm})$.
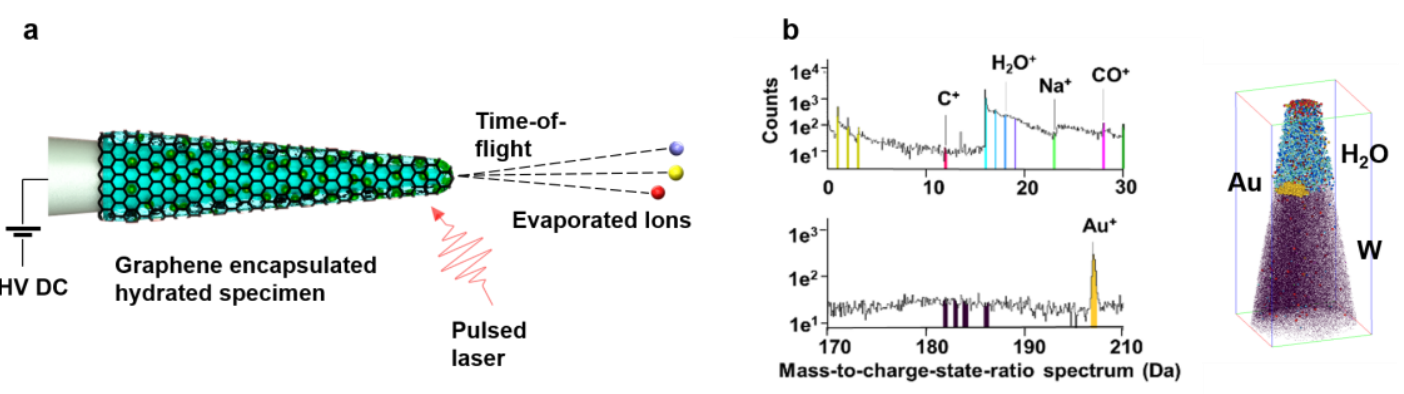

Figure 2. (a) Schematic of probing the graphene-encapsulated gold nanoparticle (AuNP) specimen. (b) Mass-to-charge-state ratio spectrum and the 3D chemical map of the field-evaporated volume containing a partial AuNP [3].

\section{References}

[1] S. Qiu, V. Garg, S. Zhang, Y. Chen, J. Li, A. Taylor, R.K.W. Marceau, J. Fu, Graphene encapsulation enabled high-throughput atom probe tomography of liquid specimens, Ultamicroscopy (2020) 113036.

[2] S. Qiu, C. Zheng, V. Garg, Y. Chen, G. Gervinskas, J. Li, M.A. Dunstone, R.K.W. Marceau, J. Fu, Three-dimensional chemical mapping of a single protein in the hydrated state with atom probe tomography, Analytical Chemistry 92(7) (2020) 5168-5177.

[3] S. Qiu, C. Zheng, Q. Zhou, D. Dong, Q. Shi, V. Garg, W. Cheng, R.K.W. Marceau, G. Sha, J. Fu, Direct Imaging of Liquid-Nanoparticle Interfaces with Atom Probe Tomography, The Journal of Physical Chemistry C 124(35) (2020) 19389-19395. 